\title{
OPEN CRISPR/Cas9-mediated base-editing enables a chain reaction through sequential repair of sgRNA scaffold mutations
}

\author{
Tsuyoshi Fukushima ${ }^{1}$, Yosuke Tanaka ${ }^{1 凶}$, Keito Adachi $^{1}$, Nanami Masuyama ${ }^{2,3}$, \\ Akiho Tsuchiya $^{1}$, Shuhei Asada ${ }^{1,4}$, Soh Ishiguro ${ }^{2,3}$, Hideto Mori ${ }^{2}$, Motoaki Seki ${ }^{2}$, \\ Nozomu Yachie ${ }^{2,3}$, Susumu Goyama ${ }^{5 \bowtie}$ \& Toshio Kitamura ${ }^{1 \bowtie}$
}

Cell behavior is controlled by complex gene regulatory networks. Although studies have uncovered diverse roles of individual genes, it has been challenging to record or control sequential genetic events in living cells. In this study, we designed two cellular chain reaction systems that enable sequential sgRNA activation in mammalian cells using a nickase Cas9 tethering of a cytosine nucleotide deaminase (nCas9-CDA). In these systems, thymidine ( $T$ )-to-cytosine (C) substitutions in the scaffold region of the sgRNA or the TATA box-containing loxP sequence (TATAloxP) are corrected by the nCas9CDA, leading to activation of the next sgRNA. These reactions can occur multiple times, resulting in cellular chain reactions. As a proof of concept, we established a chain reaction by repairing sgRNA scaffold mutations in $293 \mathrm{~T}$ cells. Importantly, the results obtained in yeast or in vitro did not match those obtained in mammalian cells, suggesting that in vivo chain reactions need to be optimized in appropriate cellular contexts. Our system may lay the foundation for building cellular chain reaction systems that have a broad utility in the future biomedical research.

A chain reaction is a sequence of related events or reactions in which each event/reaction causes the next. Since many biological processes consist of sequential chemical or cellular reactions, methods and platforms that allow molecular recording or intervention of sequential events in living cells have broad applications in cell biology, bioengineering, and therapeutics. Several systems have been developed for recording chain reactions in mammalian cells. Friedland et al. developed two cellular chain reaction systems using T7/T3 RNA polymerases and Cre/Flippase (Flp) recombinases. In the first system, T7 RNA polymerase induced the expression of T3 RNA polymerase and T3 RNA polymerase triggered the next reaction ${ }^{1}$. In the second system, recombination of the flippase recognition target (FRT) by Flp induced Cre expression, and Cre-induced LoxP recombination triggered the next reaction. Since the number of RNA polymerases and recombinases is limited, the number of chain reactions in these systems is also limited. Recently, Farzadfard et al. developed an elegant chain reaction system called $\mathrm{DOMINO}^{2}$, using a nickase Cas9 tethering of a cytosine nucleotide deaminase (nCas9-CDA), resulting in efficient substitution of the cytosine nucleotide (C) with a thymidine nucleotide $(\mathrm{T})$ in the genomic sequence ${ }^{3}$. In the DOMINO system, the $\mathrm{C}>\mathrm{T}$ modification in the target DNA locus by sgRNA and nCas9-CDA triggered the next modification. Farzadfard et al. also achieved the conversion of inactive sgRNA to active sgRNA by introducing $\mathrm{C}>\mathrm{T}$ substitutions into the protospacer sequence of nonfunctional sgRNA. One limitation of this system is the difficulty of generating multiple active sgRNAs during chain reactions because the protospacer sequence is strictly dependent on the sequence of target DNA.

To establish platforms that enable sequential activation of multiple sgRNAs, we developed two nCas9-CDAbased chain reaction systems by sequential repair of mutations in sgRNA scaffolds or TATAloxP sequences. We generated PAM-inserted sgRNAs without loss of function and successfully inactivated them by introducing $\mathrm{T}>\mathrm{C}$

\footnotetext{
${ }^{1}$ Division of Cellular Therapy, The Institute of Medical Science, The University of Tokyo, Minato-ku, Tokyo 108-8639, Japan. ${ }^{2}$ Research Center for Advanced Science and Technology, The University of Tokyo, Tokyo 153-8904, Japan. ${ }^{3}$ School of Biomedical Engineering, The University of British Columbia, Vancouver, BC V6T 1Z3, Canada. ${ }^{4}$ The Institute of Laboratory Animals, Tokyo Women's Medical University, Tokyo, Japan. ${ }^{5}$ Division of Molecular Oncology, Graduate School of Frontier Sciences, The Institute of Medical Science, The University of Tokyo, Minato-ku, Tokyo 108-8639, Japan. ${ }^{\varpi}$ email: ytims@ims.u-tokyo.ac.jp; goyama@edu.k.u-tokyo.ac.jp; kitamura@ims.u-tokyo.ac.jp
} 

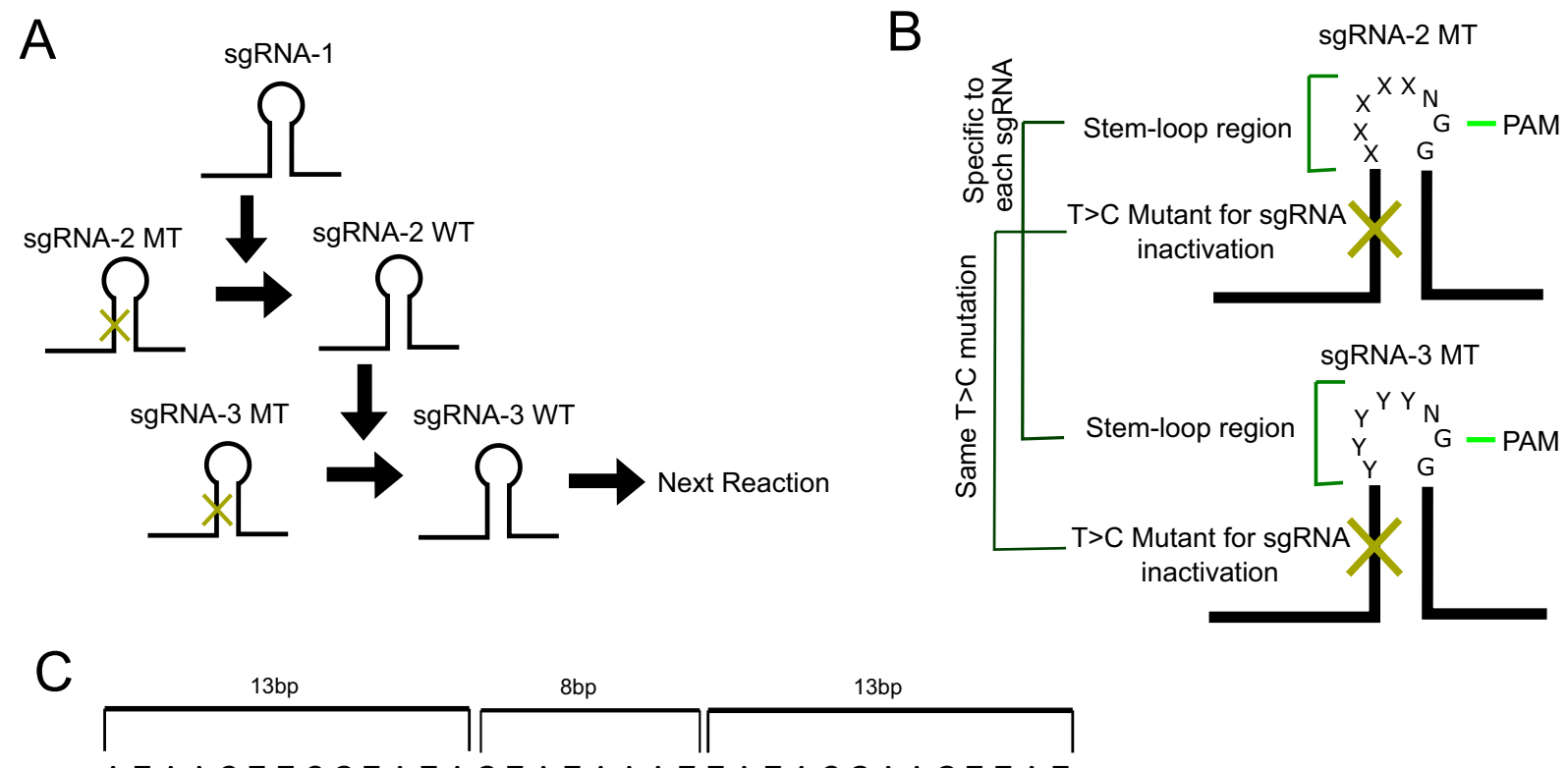

A TAACTTCGTATAGTATAAATTATACGAAGTTAT

D Transcription SgRNA-1

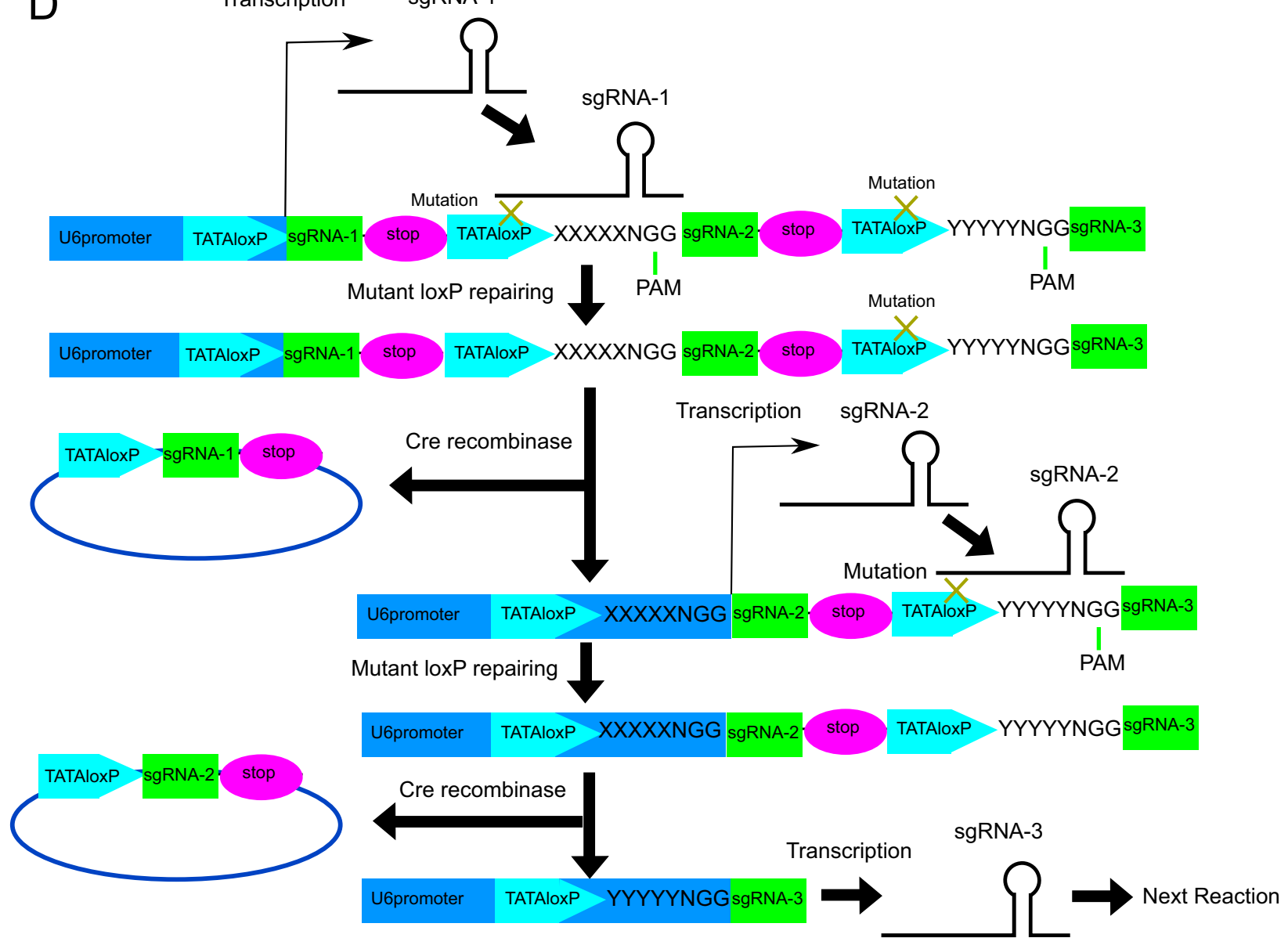

Figure 1. Screening of inactive sgRNAs with scaffold mutations in yeast. (A) Schematic representation of the chain reaction with nCas9-CDA and sgRNAs with scaffold mutations. (B) Schematic representation of the sgRNA structure with the $\mathrm{T}>\mathrm{C}$ mutation and specific sequences in the stem-loop region. (C) Schematic representation of TATAloxP structure and sequences. (D) Schematic representation of chain reaction with nCas9-CDA and TATAloxP with $\mathrm{T}>\mathrm{C}$ mutations. 
A

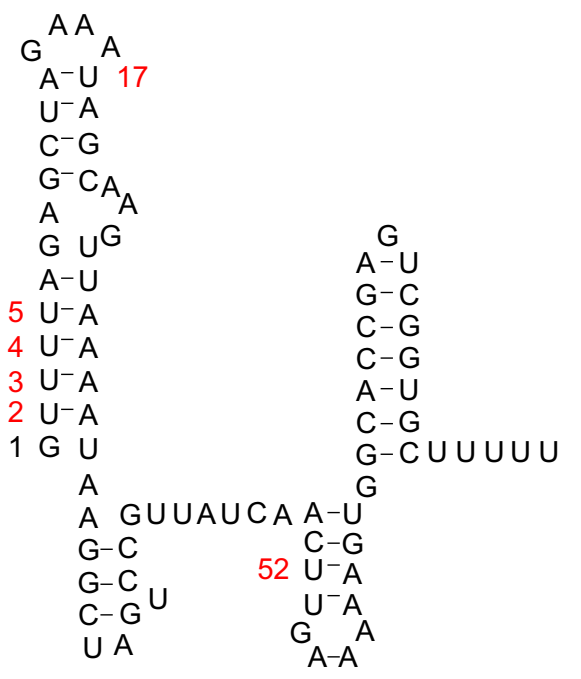

D

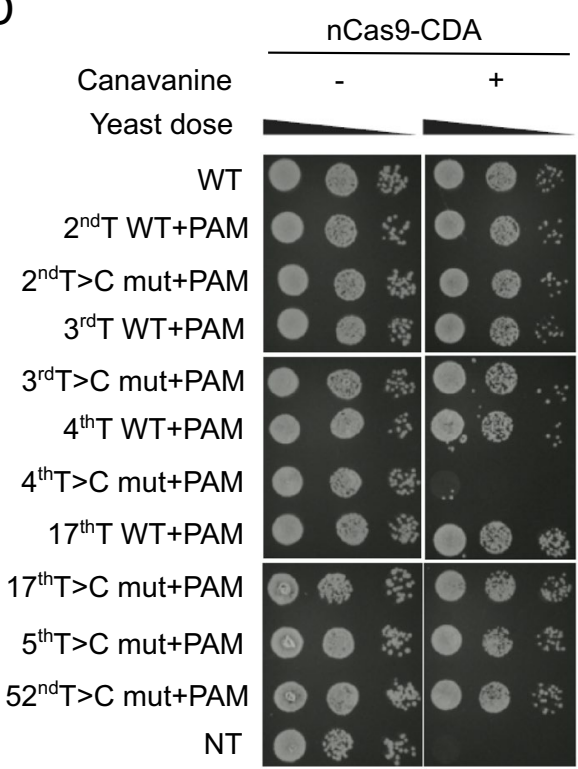

B

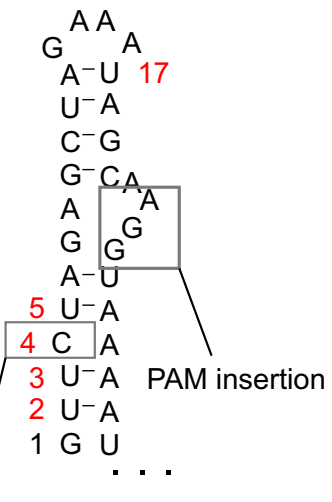

$\mathrm{T}>\mathrm{C}$ Mutantation
C

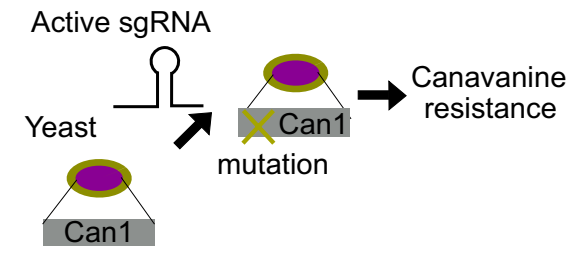

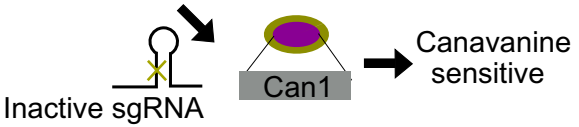

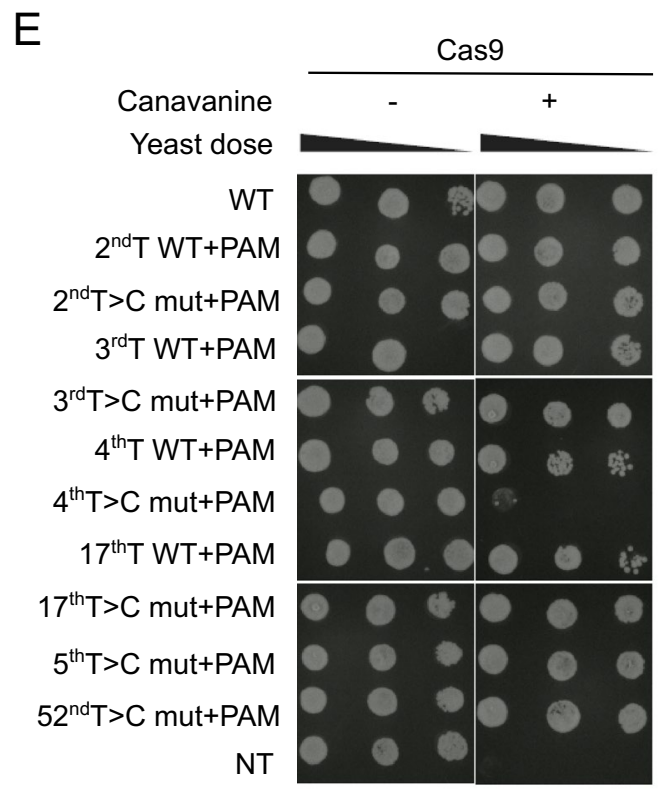

Figure 2. Screening of inactive sgRNAs with scaffold mutations in yeast. (A) Schematic representation of the scaffold region of sgRNAs. (B) Schematic representation of part of the scaffold region of sgRNAs with T $>C$ mutations and PAM insertion. (C-E) Schematic representation $(\mathbf{C})$ and results of the canavanine assay with nCas9-CDA (D) or Cas9 (E). Yeasts transduced with the indicated sgRNA were grown on plates with (right column) or without (left column) canavanine.

mutations that can be repaired by nCas9-CDA. As a proof of concept, we established a cellular chain reaction by repairing sgRNA scaffold mutations in 293 T cells. Our system could form the basis for the construction of sophisticated chain reaction systems in living cells.

\section{Results}

Two strategies to generate cellular chain reactions using nCas9-CDA. We designed two systems to generate chain reactions using nCas9-CDA. In the first system, the $\mathrm{T}>\mathrm{C}$ mutations are introduced in the scaffold region of sgRNA to disrupt its structure. First sgRNA without mutation (sgRNA-1) and nCas9-CDA correct the $\mathrm{T}>\mathrm{C}$ mutations in the second sgRNA ( $g$ RNA-2). The corrected sgRNA-2 in turn corrects the $\mathrm{T}>\mathrm{C}$ mutations in the third sgRNA (sgRNA-3) in cooperation with nCas9-CDA (Fig. 1A). Because the sequence and the length of the stem-loop region in the sgRNA scaffold are not restrictive ${ }^{4,5}$, we can design multiple sgRNAs by introducing various sequences in the stem-loop region (XXXXX in sgRNA-2-MT, YYYYY in sgRNA-3-MT) (Fig. 1B).

In the second system, the $\mathrm{T}>\mathrm{C}$ mutations are introduced in the TATAloxP sequence (TATAloxP-MT). TATAloxP forms a 13-8-13 structure, which is composed of an 8-bp spacer sequence containing TATA box flanked on 
A

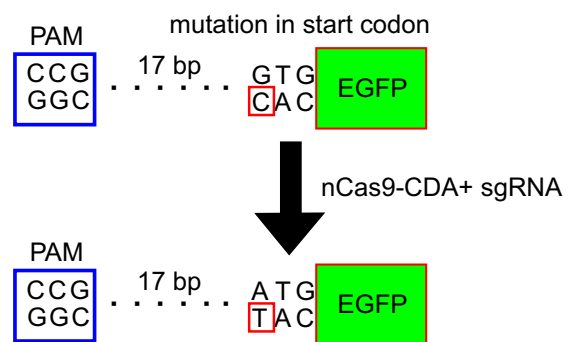

Repairing start codon

C

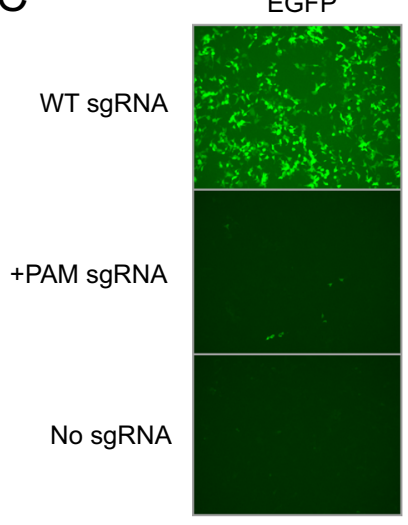

E EGFP
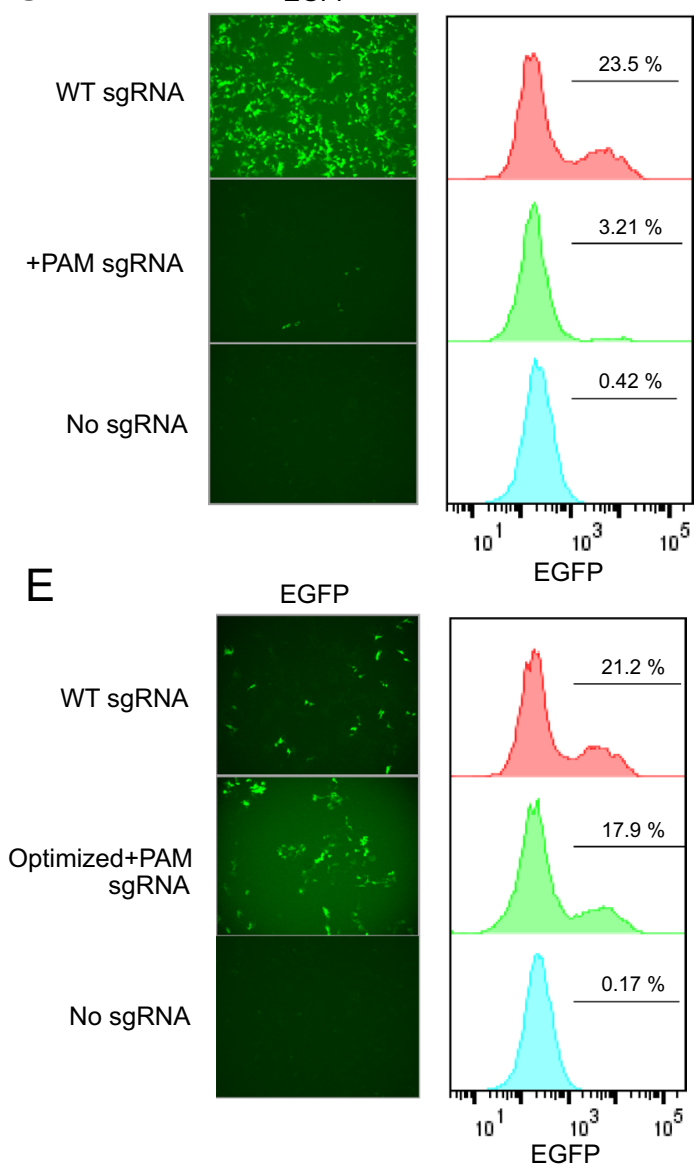

B

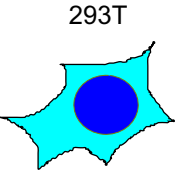

mutant EGFP expressing 293T

Lentiviral infection pLV-mutant EGFP
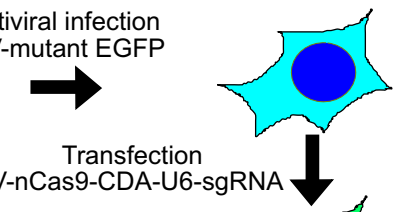

pLV-nCas9-CDA-U6-sgRNA

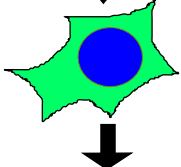

Imaging or FACS analysis

D WT ${ }_{A-U}^{A A} A$ A-U

$C-G$ G- ${ }^{-} A_{A}$ $7 \mathrm{G} \cdot U^{\mathrm{G}}$ A $-U$ $U-A$

$4 U-A$

$U-A$

$U-A$

$G \cdot U$.

+PAM

${ }_{A-U}^{A A} A$

$U-A$

C-G

${ }_{7}^{\mathrm{G}}{ }^{-}{ }_{\mathrm{G}} \mathrm{A}_{\mathrm{A}}$

$7 \mathrm{G} \mathrm{G}^{\mathrm{G}}$

$A-U$

$U-A$

$4 U-A$

$U-A$

$U-A$

G.U.

Optimized+PAM

${ }_{G-C}^{A A} A$

C-G PAM

C-G

$\mathrm{U}-\mathrm{A}$

$A-U$

C-G

${ }^{\mathrm{G}}-\mathrm{C}_{\mathrm{A}}$

$7 \mathrm{G} \cdot U^{\mathrm{G}}$

$A-U$
F

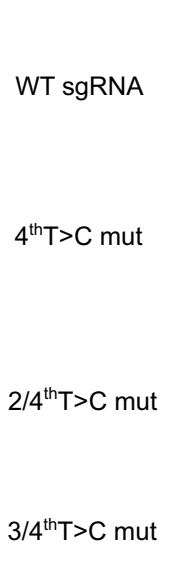

$4 / 5^{\text {th }} \mathrm{T}>\mathrm{C}$ mut

No sgRNA
EGFP

${ }^{A A} A$
$G-C$
$C-G$
$C-G$
$G-C$
$U-A$
$A-U$
$U-A$
$C-G$
$G-C A$
$A$
$G \cdot{ }^{G} A$
$A-U$
$A-U$
$U-A$
$U-A$
$U-A$
$G \cdot U$.

G.U. .

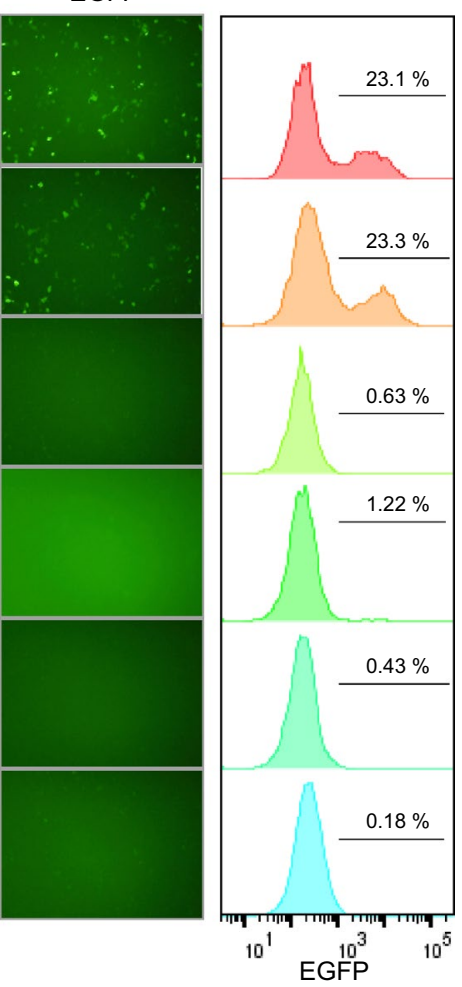


4Figure 3. Screening of inactive sgRNAs with scaffold mutations in mammalian cells. (A) Schematic representation of wild-type and mutant (ATG to GTG) EGFP with PAM sequence insertion. (B) Experimental scheme used in (C,E-F) and Fig. 4A. 293 T cells were transduced with the EGFP mutant. The mutant EGFPexpressing 293 T cells were then transfected with nCas9-CDA and sgRNAs targeting the T $>$ C mutation. (C) Fluorescence images (left) and FACS plots (right) of 293 T cells expressing mutant EGFP and nCas9-CDA together with wild-type (top), PAM-inserted (middle), or no (bottom) sgRNA. (D) Schematic representation of wild-type (left) and PAM-inserted sgRNAs (original version: middle, optimized version: right). Note that the G-U interaction at the 7th base is lost in the original PAM-inserted sgRNA. The optimized version of the PAMinserted sgRNA contains additional sequences in the stem-loop region to preserve the G-U interaction at the seventh base. (E) Fluorescence images (left) and FACS plots (right) of 293 T cells expressing mutant EGFP and nCas9-CDA together with wild-type (top), PAM-inserted (middle), or no (bottom) sgRNA. (F) Fluorescence images (left) and FACS plots (right) of 293 T cells expressing mutant EGFP and nCas9-CDA together with different sgRNAs. The indicated $\mathrm{T}>\mathrm{C}$ mutations were introduced into the control sgRNA. mut; mutation.

either side by two 13-bp inverted repeats (Fig. 1C). Cre recombinase binds to the 13 bp repeats of the TATAloxP, and importantly, this TATA box containing sequence can be incorporated into the U6 promoter to induce sgRNA expression $^{6}$. The $\mathrm{T}>\mathrm{C}$ mutations in the TATAloxP are corrected by the nCas9-CDA and the sgRNA (sgRNA-1) targeting the mutations and adjacent sequences (XXXXX in Fig. 1D). After the correction, a loxP flanked stop cassette is excised by Cre recombinase, which drives expression of the second sgRNA (sgRNA-2). The sgRNA-2 targets the next mutation and adjacent sequences (YYYYY in Fig. 1D), resulting in expression of the third sgRNA (sgRNA-3) (Fig. 1D). Because there is no restriction for the adjacent sequences, we can design multiple sgRNAs with various adjacent sequences that can be activated during the chain reaction.

Generation of inactive sgRNAs with $\mathrm{T}>\mathrm{C}$ mutations in the scaffold region. To establish the first system, we introduced $\mathrm{T}>\mathrm{C}$ mutations into the template sequence of the sgRNA scaffold region to inactivate it. Because nCas9-CDA was shown to efficiently induce $\mathrm{C}>\mathrm{T}$ substitution in DNA when the cytosine was located 18 bp upstream of PAM sequence ${ }^{3}$, we also introduced PAM sequence (NGG) 18 bp downstream of the $\mathrm{T}>\mathrm{C}$ mutation in each sgRNA template (Fig. 2A,B). We then evaluated the activity of each sgRNA with the $\mathrm{T}>\mathrm{C}$ mutations and PAM insertion using the canavanine assay. Canavanine is a toxic analog of arginine and is imported into yeast cells via a transporter Can1. Therefore, expression of Cas 9 and Can1-targeting sgRNA results in depletion of Can 1 and decreases the sensitivity of yeast to canavanine (Fig. $2 \mathrm{C})^{7}$. We used this assay to evaluate the effects of each $\mathrm{T}>\mathrm{C}$ mutation on sgRNA inactivation and found that the $\mathrm{T}>\mathrm{C}$ mutation at the fourth base resulted in sgRNA inactivation in yeast. Insertion of the PAM sequence did not inhibit sgRNA function in yeast (Fig. 2D,E).

We then examined the effects of $\mathrm{T}>\mathrm{C}$ mutations on sgRNA function in mammalian cells. An EGFP mutant was used in which the start codon was mutated from ATG to $\mathrm{GTG}^{8}$. In this EGFP mutant, the template strand has a $\mathrm{T}>\mathrm{C}$ mutation at the start codon and a PAM sequence at its $18 \mathrm{bp}$ downstream. Therefore, nCas9-CDA and active sgRNA can correct the T $>C$ mutation in the EGFP mutant and induce EGFP expression (Fig. 3A,B). However, the sgRNA did not work when a PAM sequence was inserted into the scaffold region of sgRNA, in contrast to the results of the canavanine assay (Fig. 3C). Because the weak interaction between $G$ and $U$ is known to be important for the maintenance of RNA structure ${ }^{9}$, we suspected that the loss of G-U interaction at the seventh base caused by PAM insertion might affect sgRNA function (Fig. 3D). Therefore, we introduced additional sequences near the PAM sequence to maintain the G-U interaction at the 7 th base, because extending the stem-loop sequences in the sgRNA scaffold does not affect sgRNA function ${ }^{4,5}$. As expected, this optimized PAM-inserted sgRNA maintained the G-U interaction at the 7th base (Fig. 3D) and efficiently induced EGFP expression when expressed with nCas9-CDA and mutant EGFP in $293 \mathrm{~T}$ cells (Fig. 3E). We next examined whether the $\mathrm{T}>\mathrm{C}$ mutation at the 4th base, which decreased sgRNA activity in yeast, also inactivated sgRNA in mammalian cells. Again, we obtained different results in mammalian cells from those in the canavanine assay. The sgRNA with a single $\mathrm{T}>\mathrm{C}$ mutation at the fourth base did not inactivate GFP expression, indicating that it retained its normal function. Therefore, in addition to the 4 th base, we introduced $\mathrm{T}>\mathrm{C}$ mutations at 2,3 , or 5 bases into the sgRNA template. These sgRNAs with double $\mathrm{T}>\mathrm{C}$ mutations at $2 / 4$ th, $3 / 4$ th, $4 / 5$ th bases lost the function to restore GFP expression in $293 \mathrm{~T}$ cells (Fig. 3F). We then performed similar experiments with the PAM-inserted sgRNAs and confirmed that the double $\mathrm{T}>\mathrm{C}$ mutations at $3 / 4$ bases, but not the single $\mathrm{T}>\mathrm{C}$ mutation at the 4 th base, resulted in loss of sgRNA function (Fig. 4A). Thus, we generated an inactive sgRNA with $\mathrm{T}>\mathrm{C}$ mutations and PAM sequence in the scaffold region whose function can theoretically be restored by nCas9-CDA-induced base editing in mammalian cells.

Establishment of a chain reaction by repair of sgRNA scaffold mutations. Next, we investigated whether the inactive sgRNA with $\mathrm{T}>\mathrm{C}$ mutations can be converted to the active form by nCas9-CDA-induced base editing in mammalian cells. First, we transduced nCas9-CDA, the EGFP mutant, a PAM-inserted control or inactive sgRNA targeting the EGFP mutation, with or without the second sgRNA in $293 \mathrm{~T}$ cells. The second sgRNA was designed to correct the $\mathrm{T}>\mathrm{C}$ mutations in the EGFP-targeting sgRNA and restore it to an active form (Fig. 4B,C). As expected, the optimized version of the PAM-inserted sgRNA efficiently corrected the EGFP mutation and induced GFP expression, whereas the version with $\mathrm{T}>\mathrm{C}$ mutation did not. Importantly, coexpression of the second sgRNA restored the function of the inactive sgRNA and resulted in robust GFP expression in 293 T cells (Fig. 4D). Thus, the chain reaction was successfully established in mammalian cells with nCas9-CDA and two sgRNAs targeting the $\mathrm{T}>\mathrm{C}$ mutation or EGFP mutation. 
A Optimized+PAM sgRNA

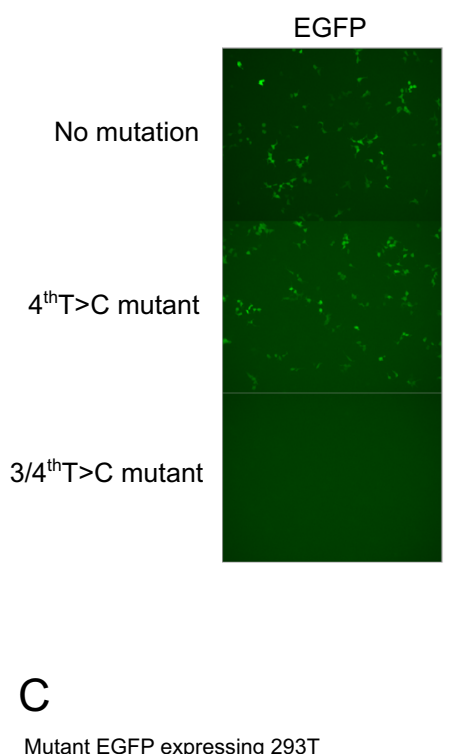

Mutant EGFP expressing 293T
B

T>C mutation-targeting sgRNA (sgRNA-1)
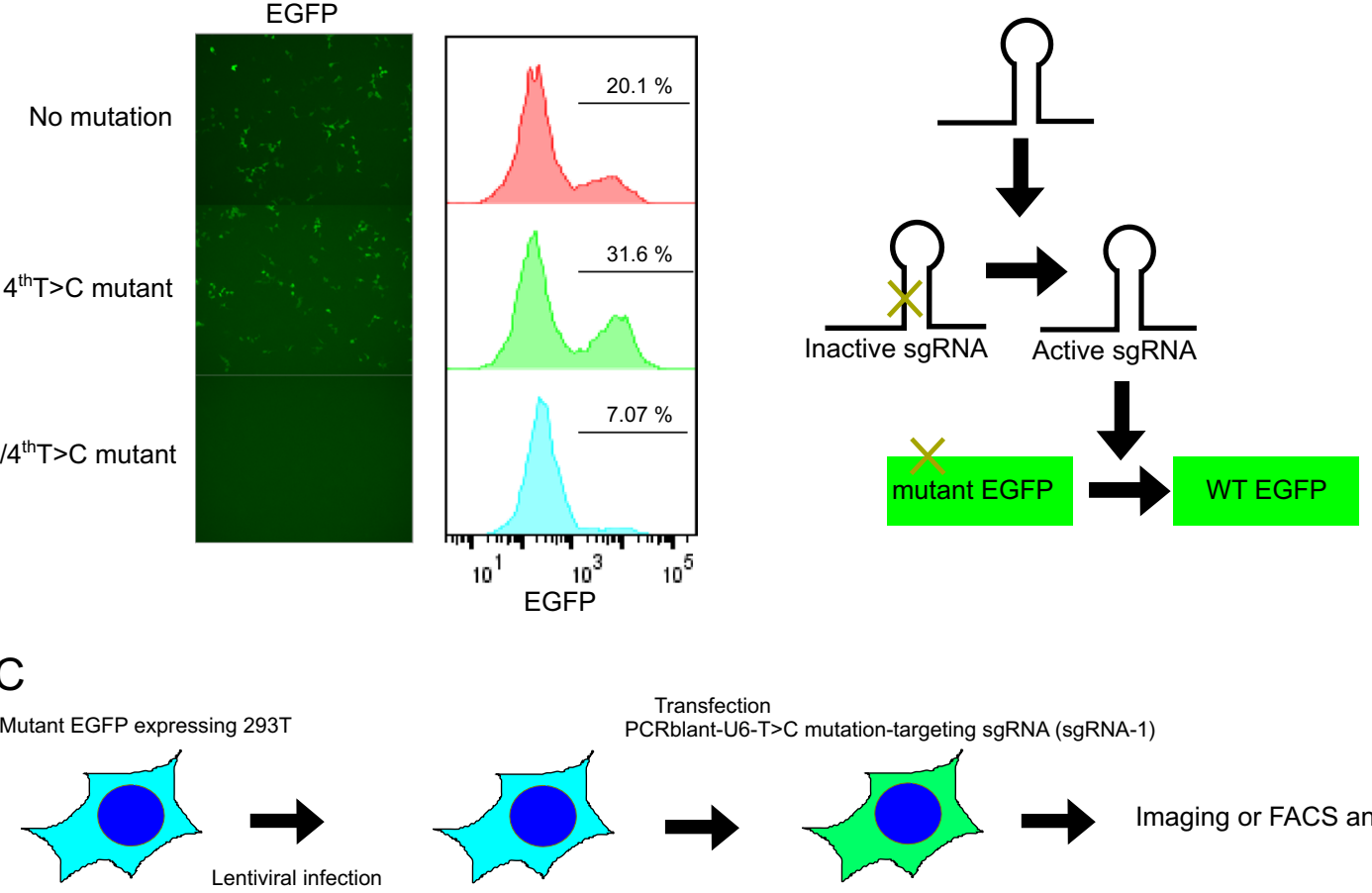

Transfection PCRblant-U6-T>C mutation-targeting sgRNA (sgRNA-1)

pLV-nCas9-CDA-U6-EGFP-targeting sgRNA (sgRNA-2)

$\mathrm{D}$
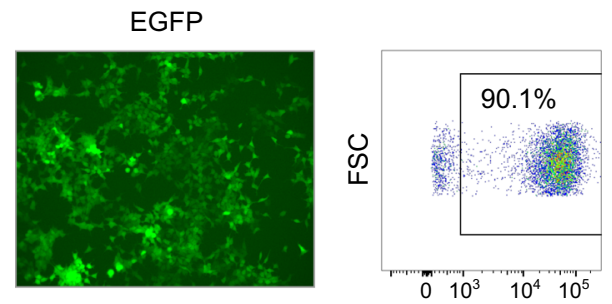

Optimized+PAM sgRNA

Optimized +PAM sgRNA with 3rd/4th $T>C$ mutations (sgRNA-2)
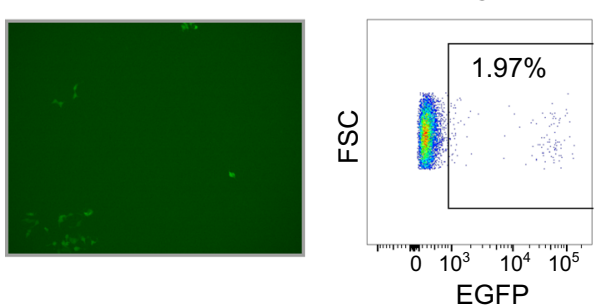

Optimized +PAM sgRNA with 3rd/4th $\mathrm{T}>\mathrm{C}$ mutations (sgRNA-2) $+$

$\mathrm{T}>\mathrm{C}$ mutation-targeting sgRNA (sgRNA-1)
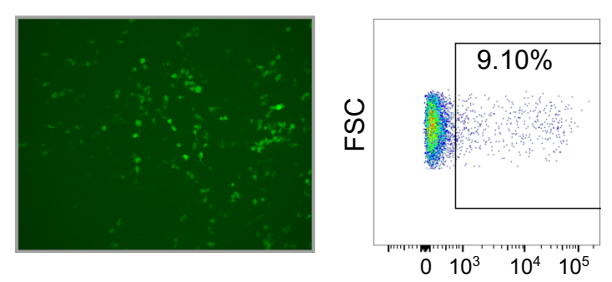

EGFP

Figure 4. Cellular chain reaction systems through sequential repair of sgRNA scaffold mutations. (A) Fluorescence images (left) and FACS plots (right) of 293 T cells expressing mutant EGFP and nCas9-CDA together with different sgRNAs. The indicated $\mathrm{T}>\mathrm{C}$ mutations were introduced into the optimized PAMinserted sgRNA. mut; mutation. (B) Schematic representation of the chain reaction by repair of sgRNA scaffold mutations to express EGFP in 293 T cells. (C) Experimental scheme as in (D). 293 T cells were transduced with mutant EGFP, nCas9-CDA and an EGFP-targeting sgRNA, then transfected with another sgRNA targeting the T > C mutation. (D) Fluorescence images (left) and FACS plots (right) of $293 \mathrm{~T}$ cells expressing mutant EGFP and nCas9-CDA together with the indicated sgRNAs. The sgRNA-1 converted the inactive sgRNA-2 to an active form, and the sgRNA-2 corrected the EGFP mutation to induce EGFP expression. 

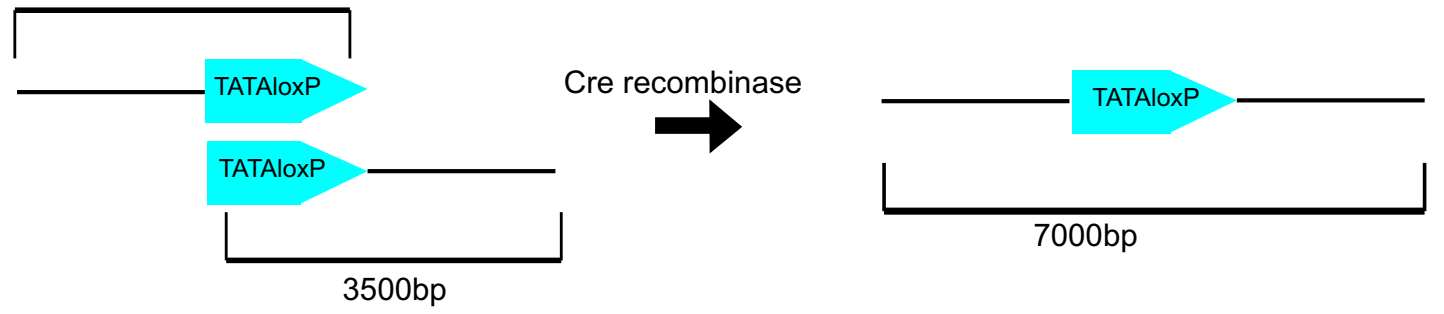

B position of $\mathrm{T}>\mathrm{C}$ mutant
Recombined DNA
Original DNA

C

NC WT $1^{\text {st }} 3^{\text {rd }} 4^{\text {th }} 11^{\text {th }} 13^{\text {th }}$ NC WT $1^{\text {st }} 3^{\text {rd }} 4^{\text {th }} 11^{\text {th }} 13^{\text {th }}$ PC

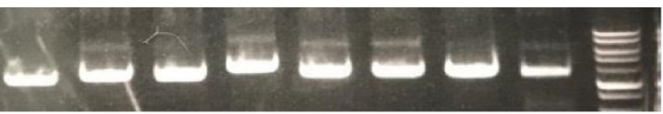

position of T>C mutant $\underset{W T}{W T} 1_{\text {st }}^{\text {st }}$

Recombined DNA Original DNA

taint

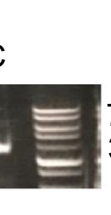

$=7000 \mathrm{~b}$

NC WT WT WT WT NC NC WT $1^{\text {st }} 13^{\text {th }}$

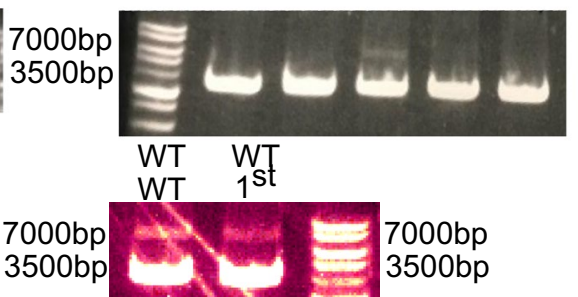

Figure 5. In vitro Screening of non-responsive TATAloxP with $\mathrm{T}>\mathrm{C}$ mutations. (A) Schematic representation of the in vitro recombination assay with TATAloxP sequences. $(\mathbf{B}, \mathbf{C})$ Linear DNAs with different TATAloxP sequences containing the indicated mutations were incubated with Cre recombinase. Note that TATAloxP with the $\mathrm{T}>\mathrm{C}$ mutation at the first base probably did not efficiently produce recombined DNA in the first experiment because of technical errors (B), but did so very well in the second experiment (C). (NC; No loxP, PC; positive control DNA in Cre recombinase (NEB Catlog\# M0298S)).

Generation of non-responsive TATAloxP sequences with T $>$ C mutations. To establish the second system, we introduced $\mathrm{T}>\mathrm{C}$ mutations into the TATAloxP sequences to make them insensitive to Cre-induced recombination. We generated several TATAloxP mutants in which a $\mathrm{T}$ base in the 13-bp repeat sequence (Fig. 1C) was replaced by C. These DNAs with different TATAloxP sequences at the $5^{\prime}$ or $3^{\prime}$ side (approximately 3500 bp-vector) were linearized and incubated with Cre recombinase. In this in vitro assay, Cre-induced recombination results in linear DNA of approximately $7000 \mathrm{bp}$ when Cre recombinase can recognize the TATAloxP sites (Fig. 5A). As shown in Fig. 5B,C, the T $>$ C mutation at the 13th base resulted in reduced recombination in both mutant/mutant and mutant/wild-type incubations.

We next investigated whether the TATAloxP sequences containing the $\mathrm{T}>\mathrm{C}$ mutations are resistant to Cremediated recombination in mammalian cells. We generated an expression vector containing a polyA signal flanked by two TATAloxP sites and a downstream EGFP cassette under the EF1a promoter in which one of the TATAloxP sites has the $\mathrm{T}>\mathrm{C}$ mutations. If the mutated TATAloxP site is resistant to Cre-mediated recombination, EGFP is not expressed even in the presence of Cre recombinase (Fig. 6A). We expressed the TATAloxP (wild-type or mutant)-EGFP constructs in $293 \mathrm{~T}$ cells together with Cre-R32V, a mutant Cre recombinase with improved fidelity ${ }^{10}$ (Fig. 6B). However, in contrast to the results of the in vitro experiments, the $\mathrm{T}>\mathrm{C}$ mutation at 13 base did not prevent Cre-R32V-induced EGFP expression in either or both 13-bp arms (Fig. 6C,D). Thus, the T>C mutation at the 13th base was not sufficient to render the TATAloxP sequence insensitive to Cre recombinase in mammalian cells. Because a previous report showed that double mutations in both 13-bp arms, particularly at the 7 th, 8 th, 11 th, 12 th, and 13th bases efficiently disrupted the loxP structure ${ }^{11}$, we subsequently examined the effect of double $\mathrm{T}>\mathrm{C}$ mutations at the 11th and/or 13th bases on their responsiveness to Cre recombinase. Among the different combinations, we found that double $\mathrm{T}>\mathrm{C}$ mutations at the 11th and 13th bases became resistant to Cre-induced EGFP expression in both arms (Fig. 6E,F,G). In this way, we generated a TATAloxP mutant which does not respond to Cre but will resume the responsiveness to Create by nCAS9-CDA-induced base editing in mammalian cells.

Inefficient repair of TATAloxP mutations in the chain reaction system. Finally, we examined whether we could establish the cellular chain reaction system using the TATAloxp mutant. We transduced wildtype and nonresponsive TATAloxP-EGFP, Cre-R32V, and nCas9-CDA into $293 \mathrm{~T}$ cells with or without the second sgRNA. The second sgRNA was designed to correct the $\mathrm{T}>\mathrm{C}$ mutations in the nonresponsive TATAlox $\mathrm{P}$ and convert it to a responsive form (Fig. $7 \mathrm{~A}, \mathrm{~B}$ ). Consistent with previous results, Cre-R32V induced recombination of only the wild-type TATAloxP but not the mutant TATAloxP to induce EGFP expression in $293 \mathrm{~T}$ cells (Fig. 7C). Unfortunately, coexpression of the second sgRNA failed to restore EGFP expression, indicating that the TATAloxP mutant with double $\mathrm{T}>\mathrm{C}$ mutations at bases 11 and 13 is resistant to nCas9 DNA-mediated base editing. Thus, this TATAloxP system in its current form is not suitable for cellular chain reactions. 
A

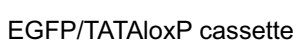

Mutation Cre recombinase

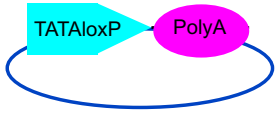

B

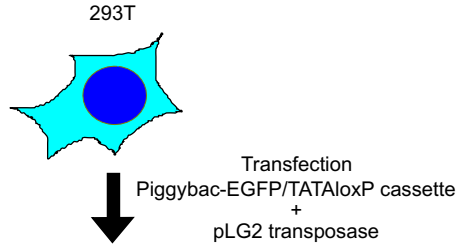

EGFP/TATAloxP cassette expressing 293T

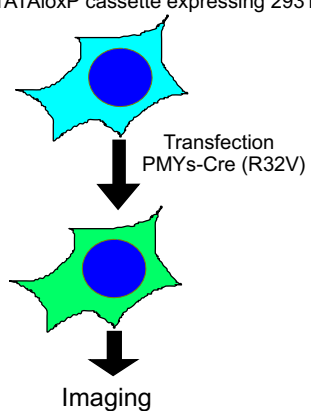

C

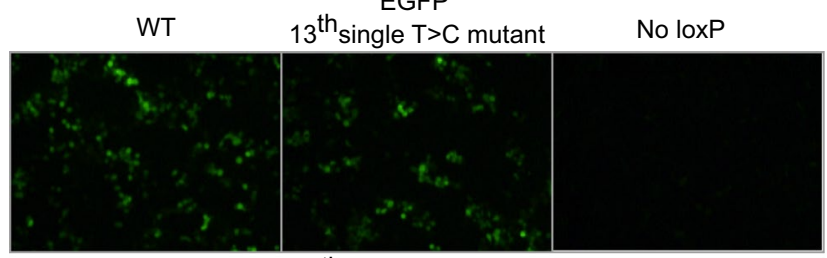

$\mathrm{D}$

WT

$13^{\text {th }}$ double $\mathrm{T}>\mathrm{C}$ mutant

No loxP

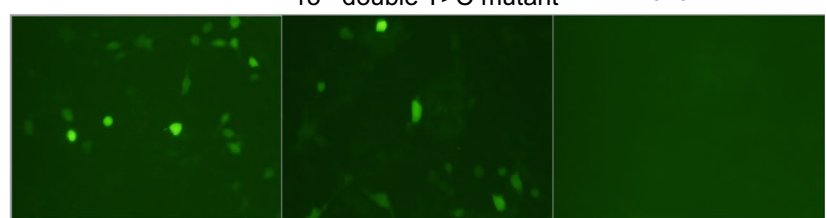

E

F

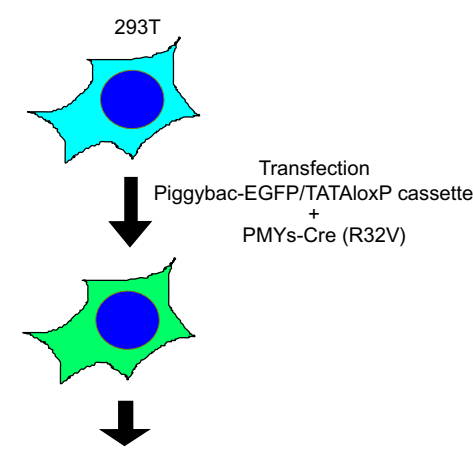

Imaging or FACS analysis

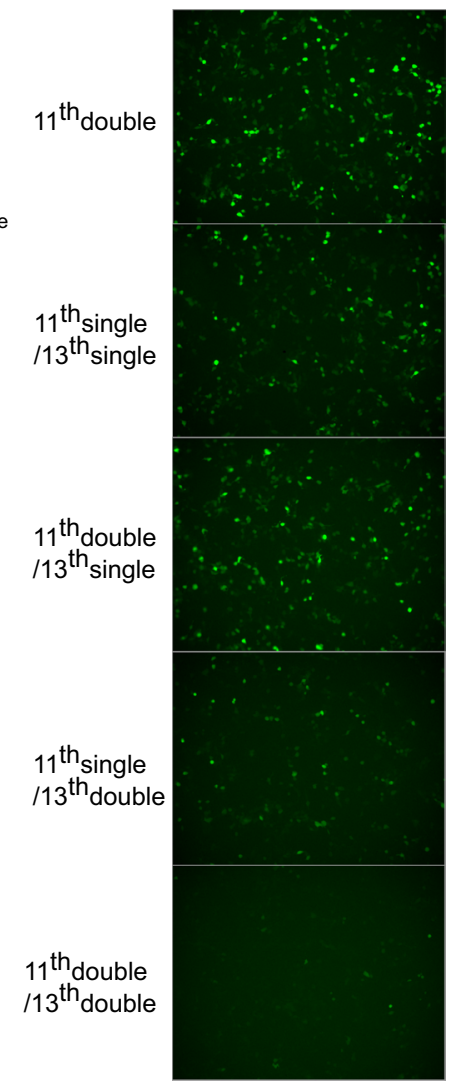

\section{G}

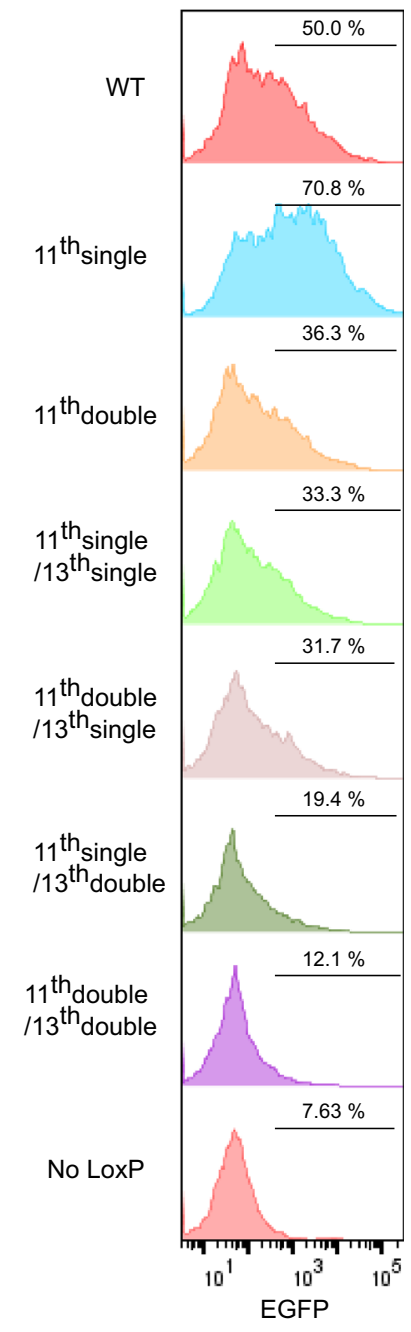

Figure 6. Screening of non-responsive TATAloxP with $\mathrm{T}>\mathrm{C}$ mutations in mammalian cells. (A) Schematic representation of the EGFP cassette with mutated TATAloxP and polyA sequences under the EF1a promoter. (B) Experimental scheme used in (C,D). $293 \mathrm{~T}$ cells were transduced with the EGFP/TATAloxP cassette, and the mutant EGFP-expressing $293 \mathrm{~T}$ cells were subsequently transfected with Cre-R32V. (C,D) Fluorescence images of $293 \mathrm{~T}$ cells expressing EGFP/ various TATAloxP with the indicated T $>\mathrm{C}$ mutations and Cre-R32V. (E) Experimental scheme as in (F,G). 293 T cells were transfected with EGFP/TATAloxP cassette and Cre-R32V. $(\mathbf{F}, \mathbf{G})$ Fluorescence images $(\mathbf{F})$ and FACS plots $(\mathbf{G})$ of 293 T cells expressing EGFP/various TATAloxP with the indicated $\mathrm{T}>\mathrm{C}$ mutations and Cre-R32V. WT; wild type. 
A
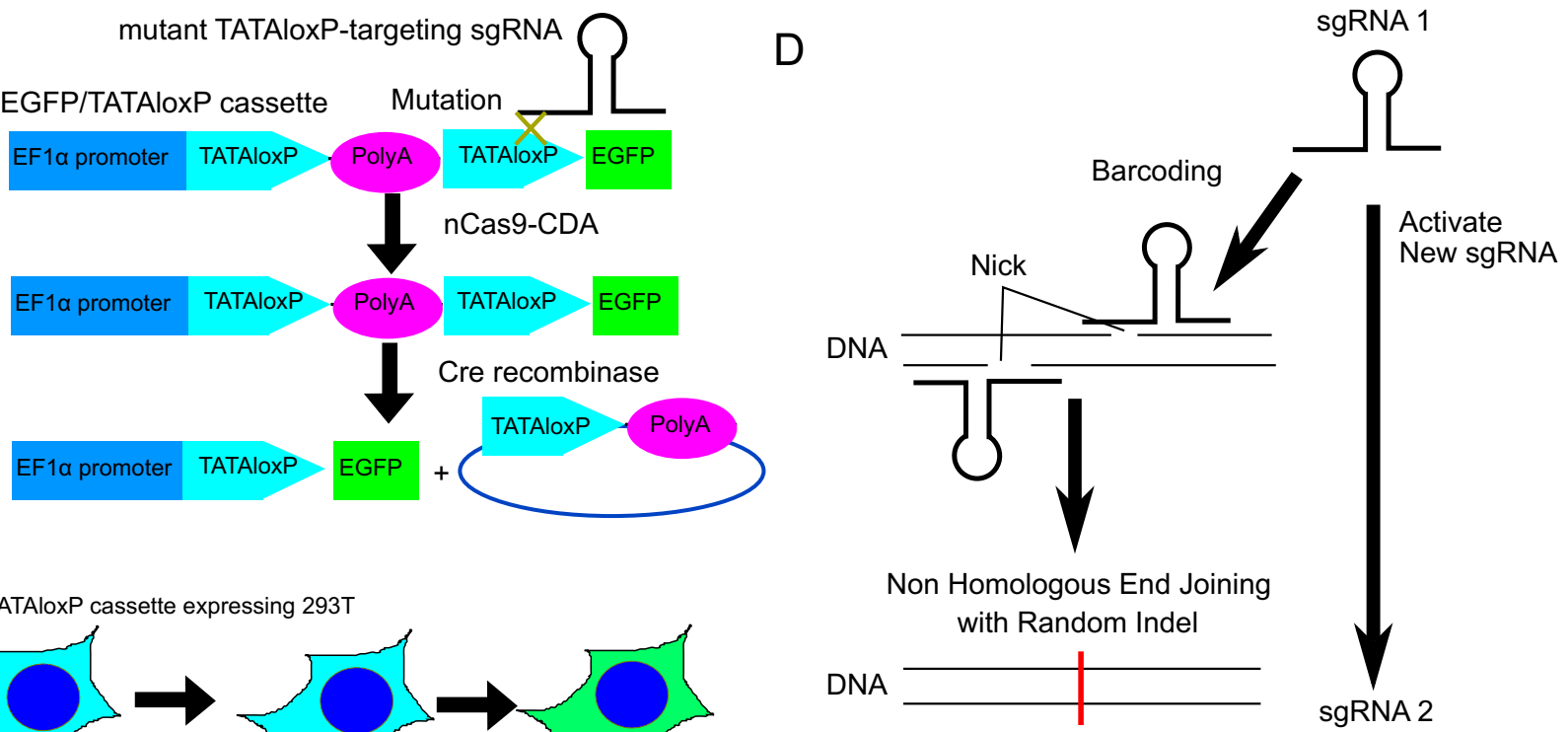

$B$

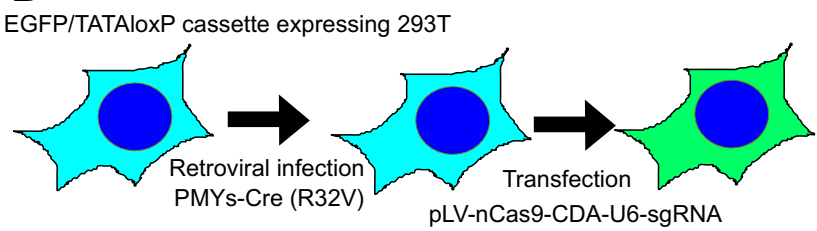

C
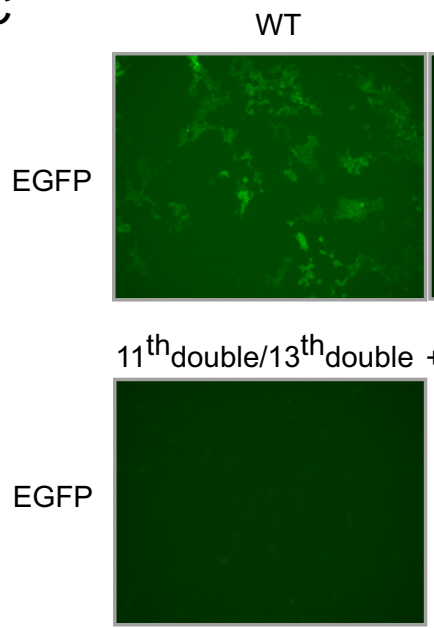

No loxP

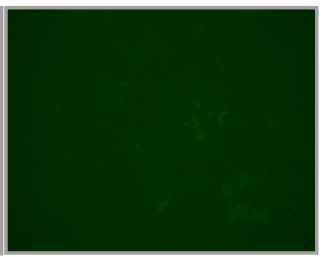

$11^{\text {th }}$ double $/ 13^{\text {th }}$ double +TATAloxP-targeting sgRNA

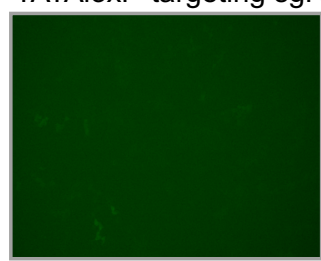

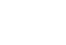

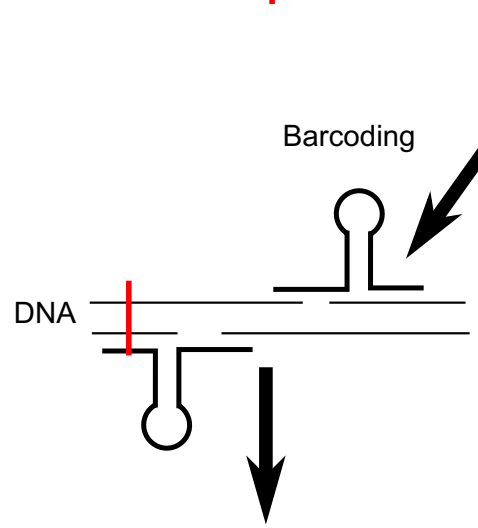

Non Homologous End Joining with Random Indel

DNA

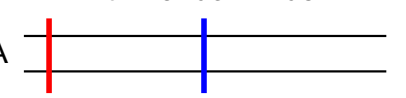

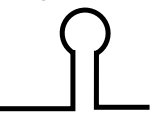

Activate New sgRNA

Figure 7. Cellular chain reaction systems through sequential repair of loxP mutations. (A) Schematic representation of the chain reaction by repair of TATAloxP mutations to express EGFP in 293 T cells. (B) Experimental scheme used in (C). $293 \mathrm{~T}$ cells were transduced with the EGFP/different TATAloxP cassette with the indicated $\mathrm{T}>\mathrm{C}$ mutations, then infected with Cre-R32V, and then transfected with nCas9-CDA and sgRNA targeting the $\mathrm{T}>\mathrm{C}$ mutation. (C) Fluorescence images of $293 \mathrm{~T}$ cells expressing EGFP/TATAloxP cassette, Cre$\mathrm{R} 32 \mathrm{~V}$, and nCas9-CDA along with the indicated sgRNAs. When the EGFP/TATAloxP cassette was used with $\mathrm{T}>\mathrm{C}$ mutations at the 11th and 13th bases in both arms, no EGFP expression was detected, even in the presence of the TATAloxP-targeting sgRNA (bottom right). (D) Schematic representation of barcoding method.

\section{Discussion}

To develop a system that enables sequential sgRNA activation in mammalian cells, we designed two chain reaction systems using the $\mathrm{nCas} 9 \mathrm{CDA}$ base editor. As a proof of concept, we successfully established a chain reaction by repairing sgRNA scaffold mutations in $293 \mathrm{~T}$ cells. In our system, there is no theoretical limit to the number of sequential reactions. Moreover, our system allows the activation of different sgRNAs at each stage of the chain reaction. Thus, our chain reaction system provides a useful platform for recording and controlling molecular events in living cells. For example, in combination with the cell cycle-dependent CRISPR-Cas9 activation system ${ }^{12}$, our chain reaction system can be used to develop a counter for cell division. Previously, several methods to monitor cell division have been reported. Those using BrdU or H2B-EGFP dilution were used to distinguish cells that divided more than 4-5 times from those divided less ${ }^{13,14}$. Another method using Ki67-Cre was used to mark cells that divided at least once ${ }^{15}$. In contrast to these methods that only record a few cell divisions, our system has the potential to serially count each cell division. With such systems, we will be able to know how many times a stem cell divides to be a terminally differentiated cell. In addition, it will be interesting to combine 
the chain reaction system with the DNA barcoding technique. It is known that nCas9 can cause random indel by adding a nick in two places ${ }^{16}$. This random indel can be used as a "barcode". Therefore, combined with the cell division counting system described above, it is theoretically possible to introduce a barcode to each daughter cell at each cell division (Fig. 7D). Such systems will enable us to draw the exact cell lineage trees during tissue development, which is impossible with the current DNA barcoding systems.

However, further optimization will be required to develop more sophisticated chain reaction systems in mammalian cells. Although we achieved the chain reaction using the sgRNA scaffold mutation system, the activity of the repaired sgRNA was much weaker than that of the control sgRNA. We could not even restore the reactivity of mutant TATAloxP by nCas9-CDA-mediated base editing in mammalian cells. To achieve more robust chain reactions, it is necessary to increase the mutation repair rate at each step. In order to improve the repair efficiency of mutant sgRNAs, further screening is required for finding mutants that lose their function after only a single mutation or adjusting the sequence of the stem loop region of sgRNAs which is changeable without loss of function with checking repairing rate. Our study also showed that the results obtained in yeast or in vitro are not the same as those obtained in mammalian cells. It has been shown that physiological RNA structure depends not only on sequence but also on RNA modifications and binding proteins ${ }^{17}$. DNA methylation has also been shown to affect the efficiency of Cre-mediated recombination ${ }^{18}$. These in vivo-specific regulatory mechanisms could explain the discrepancy between the in vitro and in vivo results. Thus, our results strongly suggest that in vivo chain reactions need to be optimized in appropriate cellular contexts.

In summary, we have developed a cellular chain reaction system using nCas9 CDA-mediated base editing of sgRNA scaffold mutations. Our system, in combination with tissue-specific or time-dependent regulation, may have broad utility for future biomedical research.

\section{Methods}

Plasmids. Plasmids and primers used for plasmid construction are summarized in supplemental Table 1. Mutations were introduced by PCR with KOD FX NEO (TOYOBO), and mutants were cloned into vectors with Gibson assembly using Gibson Assembly Master Mix (NEB). The TATAloxP sequence was generated using gBlocks (IDT). For plasmid construction of pNMA001, the gRNA coding region was constructed by PCR with primers (5'-GTTTTAGAGCTAGAAATAGCAAGTTAAAATAAGGCTAGTCCGTTATCAACTTGAAAAAGT GGCACCGAGTCGGTGCTTTTTTGTTCACTGCCGTATATAGGCAG-3' and 5'-AAACTTCTCCGCAGT GAAAGATAAATGATCGTCAATTACGAAGACTGAACGTTTTAGAGCTAGAAATAGC-3'). The amplified product and the p426-SNR52p-gRNA.CAN1. Y-SUP4t (Addgene\#43,803) backbone fragment amplified by PCR using primers (5'- GATCATTTATCTTTCACTGCGG-3' and 5'-TGTTTTTATGTCTTCGAGTCATGT AATTA-3') were subsequently assembled by Gibson Assembly. For plasmid construction of pLV-U6p-gRNACMVp-nCas9-PmCDA1-UGI-2A-mCherry, the human U6 promoter and gRNA coding region were amplified by PCR from plasmid pSI-236 using primers (5'-GGGACAGCAGAGATCCAGTTATCGATGAGGGCC TATTTCCCATG-3') and (5'-GTAATTGATTACTATTAATAACTAGTAAAAAAGCACCGACTC GGT-3'). The amplified product and the pLV-CS-086 backbone fragment digested with SpeI and ClaI were subsequently assembled using Gibson Assembly.

Cell culture. 293 T cells (CRL-11268, ATCC, Manassas, VA, USA) were cultured in DMEM (Wako) containing $10 \%$ fetal bovine serum (FBS) (Biowest) at $37^{\circ} \mathrm{C}$ and $5 \% \mathrm{CO} 2.293 \mathrm{~T}$ was authenticated by short tandem repeat analyses and tested for mycoplasma contamination in our laboratory. For selection, $1 \mu \mathrm{g} / \mathrm{ml}$ puromycin (Thermo Fisher Scientific), $5 \mu \mathrm{g} / \mathrm{ml}$ blasticidin S (Thermo Fisher Scientific), and $1 \mathrm{mg} / \mathrm{ml}$ zeosin (InvivoGen) were used.

Transient transfection. Plasmids were transfected using the calcium phosphate co-precipitation method ${ }^{19}$. A mixture of $10-20 \mu \mathrm{g}$ plasmid $(10-20 \mu \mathrm{l}), 2.5 \mathrm{M} \mathrm{CaCl} 2(50 \mu \mathrm{l})$, and filtered water $(430-440 \mu \mathrm{l})$ to $2 \times \mathrm{HeBS}$ $(500 \mu \mathrm{l})$ was added to $293 \mathrm{~T}$ cells in a $10-\mathrm{cm}$ dish $\left(2.0 \times 10^{6}\right.$ cells/dish $)$ for $18 \mathrm{~h}$. To integrate the piggyback vector, $15 \mu \mathrm{g}$ of piggyback vector and $5 \mu \mathrm{g}$ of pLG2 (PB200PA-1, SBI) were co-transfected with the above plasmids.

Viral transduction. Lentiviruses and retroviruses were prepared by transient transfection in $293 \mathrm{~T}$ cells using the calcium phosphate method as described above. A mixture of plasmids ( $3 \mu \mathrm{g}$ VSVG and $10 \mu \mathrm{g}$ PAX2 for lentiviruses, $3 \mu \mathrm{g}$ RD114 and $10 \mu \mathrm{g}$ M57 for retroviruses along with $12 \mu \mathrm{g}$ viral vectors), $2.5 \mathrm{M} \mathrm{CaCl} 2(50 \mu \mathrm{l})$, and filtered water $(425 \mu \mathrm{l})$ to $2 \times \mathrm{HeBS}(500 \mu \mathrm{l})$ was added to $293 \mathrm{~T}$ cells in a $10-\mathrm{cm} \mathrm{dish}\left(2.0 \times 10^{6} \mathrm{cells} / \mathrm{dish}\right)$. After $24 \mathrm{~h}$, the medium was removed by aspiration and $10 \mathrm{ml}$ of fresh DMEM(Wako)/10\% FBS (biowest) was added. The virus-containing medium was collected $48 \mathrm{~h}$ after transfection.

In vitro Cre recombinase reaction assay. One $\mu \mathrm{g}$ of the mutant TATAloxP sequences in the zero-blant vector (Thermo Fisher Scientific) were incubated with $1.5 \mathrm{U} / \mathrm{ml} \mathrm{BamHI,} 1.0 \mathrm{U} / \mathrm{ml}$ NotI or $1.0 \mathrm{U} / \mathrm{ml} \mathrm{MluI} \mathrm{to}$ linearize them. The linear DNAs (20 ng each) were mixed with $10 \times$ Cre Recombinase Reaction Buffer, Cre Recombinase and $\mathrm{H} 2 \mathrm{O}$ and incubated at $37^{\circ} \mathrm{C}$ for $30 \mathrm{~min}$ and then at $70{ }^{\circ} \mathrm{C}$ for $10 \mathrm{~min}$.

Live cell imaging. Live cell imaging was performed using the Invitrogen EVOS FL Auto 2 Imaging System (Thermo Fisher Scientific). Phase contrast images were acquired using a $10 \times$ phase contrast objective and camera (with a resolution of $1388 \times 1040$ pixels). EGFP images were acquired using the EVOS ${ }^{\text {ma }}$ LED cube, GFP. 
Flowcytometry analysis. The $293 \mathrm{~T}$ cells were resuspended in PBS containing 2\% FBS. Cells were then analyzed using a FACS-Verse (BD Biosciences).

Canavanine assay. Saccharomyces cerevisiae strain BY4741 (MATa his $3 \Delta 0$ leu2 $\Delta 0$ met15 $\Delta 0$ ura3 $\Delta 0$ ) was used for CAN1 mutagenesis analysis of the CRISPR system. Parent strains BY4741 were grown before transformation in YPAD and then propagated in the appropriate synthetic complete media (SC) without the auxotrophic compound supplemented with the plasmids. BY4741 were spread onto the YPAD plate on day 1 . On day 3, BY4741 single colonies were harvested and cultured in $5 \mathrm{ml} \mathrm{YPAD}$ at $30^{\circ} \mathrm{C}$ overnight. On day 4 , transformation of plasmids containing a galactose-inducible modifier gene plasmid (Cas9, nCas9-CDA) with LEU2 marker and a gRNA-expressing plasmid with Ura3 marker ( $250 \mu \mathrm{g}$ each per transformation) was performed using a Frozen EZ Yeast Transformation Kit (ZYMO RESEARCH). After transformation, BY4741 were spread on SCLeu-Ura + Ade plates for selection. On day 6, single colonies were taken from each plate and cultured in $5 \mathrm{ml}$ SC-Leu-Ura-Ade at $30^{\circ} \mathrm{C}$. On day 8, the culture medium was changed to SRaffi-Leu-Ura+Ade. On the 10th day, the culture medium was replaced with SGall-Leu-Ura + Ade. On day 11, BY4741 were spread on SC-LeuUra + Ade + canavanine plates or SC-Leu-Ura + Ade plates.

\section{Data availability}

Information and inquiries about reagents can be directed to the main contact person, Yosuke Tanaka (ytims@ ims.u-tokyo.ac.jp).

Received: 2 February 2021; Accepted: 19 November 2021

Published online: 13 December 2021

\section{References}

1. Friedland, A. E. et al. Synthetic gene networks that count. Science 324, 1199-1202. https://doi.org/10.1126/science.1172005 (2009).

2. Farzadfard, F. et al. Single-nucleotide-resolution computing and memory in living cells. Mol. Cell 75, 769-780.e764. https://doi. org/10.1016/j.molcel.2019.07.011 (2019).

3. Nishida, K. et al. Targeted nucleotide editing using hybrid prokaryotic and vertebrate adaptive immune systems. Science 353, 6305. https://doi.org/10.1126/science.aaf8729 (2016).

4. Chen, B. et al. Dynamic imaging of genomic loci in living human cells by an optimized CRISPR/Cas system. Cell 155, 1479-1491. https://doi.org/10.1016/j.cell.2013.12.001 (2013).

5. Nishimasu, H. et al. Crystal structure of Cas9 in complex with guide RNA and target DNA. Cell 156, 935-949. https://doi.org/10. 1016/j.cell.2014.02.001 (2014).

6. Ventura, A. et al. Cre-lox-regulated conditional RNA interference from transgenes. Proc. Natl. Acad. Sci. USA 101, 10380-10385. https://doi.org/10.1073/pnas.0403954101 (2004).

7. DiCarlo, J. E. et al. Genome engineering in Saccharomyces cerevisiae using CRISPR-Cas systems. Nucleic Acids Res. 41, 4336-4343. https://doi.org/10.1093/nar/gkt135 (2013).

8. Sakata, R. C. et al. Base editors for simultaneous introduction of C-to-T and A-to-G mutations. Nat. Biotechnol. 38, 865-869. https://doi.org/10.1038/s41587-020-0509-0 (2020).

9. Varani, G. \& McClain, W. H. The G x U wobble base pair. A fundamental building block of RNA structure crucial to RNA function in diverse biological systems. EMBO Rep. 1, 18-23. https://doi.org/10.1093/embo-reports/kvd001 (2000).

10. Eroshenko, N. \& Church, G. M. Mutants of Cre recombinase with improved accuracy. Nat. Commun. 4, 2509. https://doi.org/10. 1038/ncomms3509 (2013).

11. Hartung, M. \& Kisters-Woike, B. Cre mutants with altered DNA binding properties. J. Biol. Chem. 273, 22884-22891. https://doi. org/10.1074/jbc.273.36.22884 (1998).

12. Matsumoto, D., Tamamura, H. \& Nomura, W. A cell cycle-dependent CRISPR-Cas 9 activation system based on an anti-CRISPR protein shows improved genome editing accuracy. Commun. Biol. 3, 601. https://doi.org/10.1038/s42003-020-01340-2 (2020).

13. Wilson, A. et al. Hematopoietic stem cells reversibly switch from dormancy to self-renewal during homeostasis and repair. Cell 135, 1118-1129. https://doi.org/10.1016/j.cell.2008.10.048 (2008).

14. Bernitz, J. M., Kim, H. S., MacArthur, B., Sieburg, H. \& Moore, K. Hematopoietic stem cells count and remember self-renewal divisions. Cell 167, 1296-1309.e1210. https://doi.org/10.1016/j.cell.2016.10.022 (2016).

15. He, L. et al. Proliferation tracing reveals regional hepatocyte generation in liver homeostasis and repair. Science 371, 6532. https:// doi.org/10.1126/science.abc4346 (2021).

16. Ran, F. A. et al. Double nicking by RNA-guided CRISPR Cas9 for enhanced genome editing specificity. Cell 154, 1380-1389. https:// doi.org/10.1016/j.cell.2013.08.021 (2013).

17. Spitale, R. C. et al. Structural imprints in vivo decode RNA regulatory mechanisms. Nature 519, 486-490. https://doi.org/10.1038/ nature14263 (2015).

18. Rassoulzadegan, M., Magliano, M. \& Cuzin, F. Transvection effects involving DNA methylation during meiosis in the mouse. EMBO J 21, 440-450. https://doi.org/10.1093/emboj/21.3.440 (2002).

19. Kitamura, T. et al. Retrovirus-mediated gene transfer and expression cloning: powerful tools in functional genomics. Exp. Hematol. 31, 1007-1014 (2003).

\section{Acknowledgements}

We thank the Center for Flow Cytometry at the Institute of Medical Science, University of Tokyo, for its help. This project was supported by "Grant-in-Aid for Challenging Exploratory Research" from JSPS to Y.T. (19K22380) and "Grant-in-Aid for Challenging Exploratory Research" from JSPS to Tsu.F. (20K21613).

\section{Author contributions}

T.F. and Y.T. planned most of the experiments. TF., K.A. and A.T performed most of the experiments. N.M. performed the canavanine assay. S.A., S.I., H.M., and M.S. helped plan the experiments. T.F., S.G., Y.T, and T.K. wrote the manuscript. Y.T., T.K. Y.N., and S.G. supervised and coordinated the project. 


\section{Competing interests}

The authors declare no competing interests.

\section{Additional information}

Supplementary Information The online version contains supplementary material available at https://doi.org/ 10.1038/s41598-021-02986-6.

Correspondence and requests for materials should be addressed to Y.T., S.G. or T.K.

Reprints and permissions information is available at www.nature.com/reprints.

Publisher's note Springer Nature remains neutral with regard to jurisdictional claims in published maps and institutional affiliations.

(c) (1) Open Access This article is licensed under a Creative Commons Attribution 4.0 International License, which permits use, sharing, adaptation, distribution and reproduction in any medium or format, as long as you give appropriate credit to the original author(s) and the source, provide a link to the Creative Commons licence, and indicate if changes were made. The images or other third party material in this article are included in the article's Creative Commons licence, unless indicated otherwise in a credit line to the material. If material is not included in the article's Creative Commons licence and your intended use is not permitted by statutory regulation or exceeds the permitted use, you will need to obtain permission directly from the copyright holder. To view a copy of this licence, visit http://creativecommons.org/licenses/by/4.0/.

(C) The Author(s) 2021 